\title{
The Spite plateau: a puzzle and a challenge for the stellar physicist
}

\author{
Jean-Paul Zahn ${ }^{1}$ \\ ${ }^{1}$ LUTH, Observatoire de Paris, F-92195 Meudon, France \\ email: jean-paul.zahn@obspm.fr
}

\begin{abstract}
In order to use the lithium abundance of the Spite plateau to constrain the Big Bang Nucleosynthesis, one has to determine how much of the original lithium has been destroyed by the various physical processes that are known to operate in stellar radiation zones. These are briefly reviewed, with emphasis on the mixing occurring in tachoclines and on that generated indirectly by the transport of angular momentum through internal gravity waves.
\end{abstract}

Keywords. Stars: abundances, stars: interiors, stars: Population II, stars: rotation, turbulence, waves

\section{Why lithium is depleted in halo stars}

We are here to happily celebrate the very important discovery made by Monique and François Spite that the oldest stars of our Galaxy have a lithium abundance of about $\mathrm{N}(\mathrm{Li})=2.05$, in the usual logarithmic units, a value which does not depend on metallicity nor on effective temperature. Assuming that this value represents the original lithium content in those stars, this observation provided one of the very few confirmations of the Big Bang Theory.

However some stellar physicists began to question this hypothesis. First because Li depletion clearly occurs in Population I stars, where it causes the well-known Li dip (Boesgaard \& Tripicco 1986). Why would the Population II stars be spared? And among those, why do the ultra-Li-poor stars break the rule (Ryan et al. 2001)?

And now, with the results of WMAP (Bennett et al. 2003, Spergel et al. 2003), nobody doubts anymore that some depletion has occurred in the plateau stars.

On the theoretical side, one knew that gravitational settling would decrease the surface abundance of any element with respect to hydrogen, whereas radiative acceleration could act in the opposite way. Noerdlinger and his collaborators were the first to assess the effect of microscopic diffusion: they applied it to helium, and showed that the settling of that element would modify the evolution of Population II stars, and hence that it would shorten the estimated age of globular clusters (Noerdlinger \& Arigo 1980; Stringfellow et al. 1883).

The first comprehensive work on lithium depletion was performed by Proffitt and Michaud (1991). They pointed out that the lithium at the surface of a star is depleted by both gravitational settling and by its transport from regions where nuclear burning occurs, and they described the whole evolution of such Population II stars. Starting from its original value, the $\mathrm{Li}$ abundance decreases rapidly due to pre-main-sequence burning at the base of the convective envelope. Then, on the main-sequence, there is a slower decrease which is caused by gravitational settling. Thereafter, as the star leaves the main-sequence, the surface abundance rises somewhat because the convective envelope begins to deepen, and dredges up some of the Li which had settled there. Finally, the 
abundance drops again, when the convective envelope reaches the region where Li has been destroyed through nuclear burning. Choosing an initial $\mathrm{Li}$ abundance $\mathrm{N}(\mathrm{Li})=2.5$, Michaud and Proffitt succeeded in fitting the Spite plateau with their diffusion models, for an age of 15 Gyr.

But they were well aware that gravitational settling is not the only process to consider when modeling element separation, and that the situation would worsen when radiative acceleration is taken into account: the $\mathrm{Li}$ abundance would then decrease with effective temperature, and the Spite plateau would no longer be a plateau (see also Deliyannis et al. 1990). Moreover, for Population I stars these microscopic processes would lead to extreme abundance anomalies in A type stars, that would greatly exceed the observed values. Such behavior can be remedied by invoking mass loss (Michaud et al. 1983, Vauclair \& Charbonnel 1995), which can be fine-tuned to reproduce the flatness of the plateau. But the most plausible explanation is that there is some mixing operating below the convection zone, which partly smoothes out the steep composition gradients due to settling and radiative acceleraton. The effect of such turbulent mixing on the surface abundances of metal-poor stars was explored in detail by Richard et al. (2002). They took both gravitational settling and radiative acceleration into account, and their mixing was parametrized by a turbulent diffusivity decreasing with depth. By adjusting the strength of that turbulent mixing, and in particular its dependence on temperature, they were able to fit the Spite plateau $\mathrm{N}(\mathrm{Li})$ vs. $\mathrm{T}_{\text {eff }}$ at 13.5 Gyr. They estimated the Li depletion to be of 0.17 dex.

\section{Beyond parametrization: rotational mixing}

Despite its success in reproducing the Spite plateau, using an ad hoc prescription for the mixing below the convection zone is hardly a satisfying solution, especially when it links the level of turbulence, which is a dynamical process, to the temperature, a thermodynamic variable. One clearly wants to go beyond that treatment, and introduce the physical processes which are responsible for the mixing in stable radiation zones.

These are well known: the main cause of mixing is the rotation of the star. First, because it generates a thermally driven meridional circulation, which transports matter and angular momentum, and thus produces differential rotation. That differential rotation, in turn, is liable to shear instabilities, which lead to turbulence. This combination of large-scale advection and turbulent diffusion is what we call 'rotational mixing'

In the original treatment (Eddington 1925, Vogt 1925, Sweet 1950), the meridional circulation was linked to the state of rotation, i.e. the circulation speed and hence the mixing scaled proportionally to the square of the angular velocity. Since such mixing was found extremely efficient in fast rotators, preventing massive stars from evolving to the giant branch, Mestel (1953) invoked the choking effect of composition gradients, an idea which was recently revived by S. Vauclair (1999; see also Théado \& Vauclair 2001). The present view is more subtle (Busse 1982, Zahn 1992). In stars which lose little angular momentum or none, there is no need to transport angular momentum to the surface; then the circulation would vanish altogether, if it had not to compensate a weak turbulent transport down the gradient of the angular velocity. Models of massive stars that include this type of rotational mixing, which has been formulated in Zahn (1992) and Maeder \& Zahn (1998), are in good agreement with observed properties, such as the presence at the surface of elements that have been synthesized in the nuclear core (Talon et al. 1997), or the ratio between red and blue supergiants in stellar clusters (Maeder \& Meynet 2000).

Until very recently, the modeling of solar-type stars has been less successful. In those stars, which lose most of their angular momentum through a wind, the meridional 


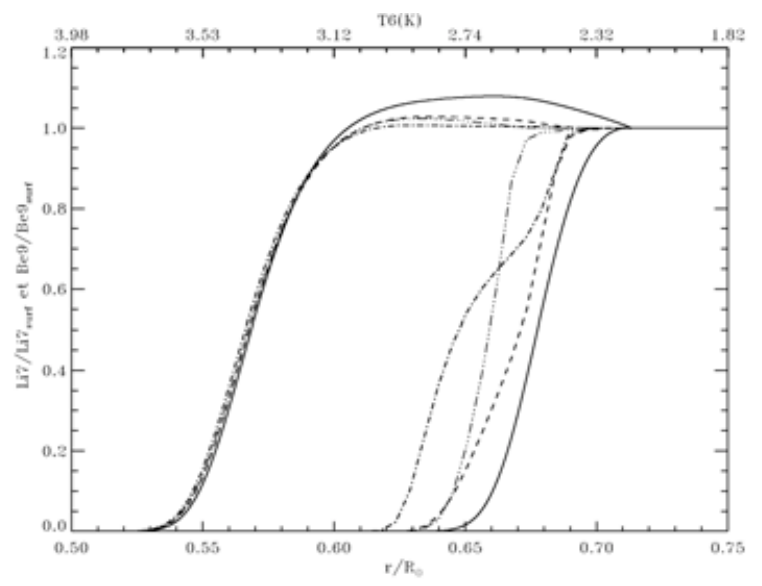

Figure 1. Radial profiles of ${ }^{7} \mathrm{Li}$ and ${ }^{9} \mathrm{Be}$, normalized to the surface value, for a solar model including only microscopic diffusion (solid line) and for 3 models where macroscopic mixing in the tachocline has been added. Note that the mixing modifies the Li profile but leaves the Be profile unaffected (Brun et al. 1999, courtesy ApJ).

circulation adjusts such as to carry the required angular momentum towards the surface, at least in the absence of other processes (Zahn 1992). One would then expect that the amount of mixing, and hence the depletion of light elements, be proportional to the loss of angular momentum. This would have as consequence that tidally-locked binaries would be less Li depleted than single stars, since the angular momentum carried away by their winds would be drawn from their orbit, not from their internal rotation; hence there would be no need to transport angular momentum inside such stars, and their Li would be preserved (Zahn 1994). However, there is no sign that tidally-locked binaries are less Li-depleted than single stars, as was shown by Balachandran (2002); hence one must conclude that the transport of matter is not correlated with the transport of angular momentum.

Another hint on that is the fact that lithium is seriously depleted in the Sun, and that beryllium is not, as was shown by Balachandran and Bell (1997), who took into account a missing UV opacity source in their spectroscopic analysis. This contrasted behavior may be explained if the mixing occurs only in the vicinity of the convection zone, i.e. in the tachocline.

\section{Tachocline mixing}

The solar tachocline is that thin region, detected through helioseismology, where the rotation changes from differential in the convection zone to quasi uniform in the radiative interior. The thinness of that layer remains a puzzle, because the differential rotation applied on the top of the radiation zone should have spread much deeper than observed, due to radiative diffusion (Spiegel \& Zahn 1992). In any case, that layer will be mixed by slow meridional flows and shear-induced turbulence, much like the bulk of the radiation zone. The difference here is that this circulation is localized in a thin region, and that because of its octopolar shape it does not transport a net amount of angular momentum. Moreover, in presence of strongly anisotropic turbulence, which is here expected because of the vertical stratification, the advective transport of chemicals is changed into a much slower vertical diffusion (Chaboyer \& Zahn 1992). 
The mixing of chemicals in the tachocline has been calculated by Brun et al. (1999), based on the Spiegel \& Zahn model: the radial profiles of ${ }^{7} \mathrm{Li}$ and ${ }^{9} \mathrm{Be}$ are displayed in fig. 1. As may be expected from the thinness of the tachocline, the profile of $\mathrm{Be}$ is hardly affected, and this element is depleted only by about $10 \%$. On the contrary, the $\mathrm{Li}$ profile is shifted toward the interior, and on the surface $\mathrm{Li}$ is depleted respectively by a factor of 8 (no mixing, continuous line), 12 (dashed line), 91 (dash-dotted) and 89 (dash-triple-dotted line), depending on the parameters chosen for the model.

We may thus conclude that the difference in $\mathrm{Li}$ and $\mathrm{Be}$ depletion observed in the Sun can be accounted for by such localized tachocline mixing. However the model described here involves two unknown parameters: one characterizing the stratification (the buoyancy frequency) and the other the horizontal component of the turbulent transport, and only one observational constraint is available, namely the present thickness of the tachocline. Moreover, the slowness of the core's rotation rate in the Sun is another puzzle of stellar evolution which cannot be ascribed to the tachocline.

\section{Internal gravity waves}

In the absence of other processes, the core of solar-type stars would rotate much more rapidly than the surface (Pinsonneault et al. 1989), unlike what we see in the Sun, thanks to helioseismology. The observed uniform rotation in the solar radiative interior can only be achieved if another process operates there, which transports angular momentum much more efficiently than meridional circulation and shear-induced turbulence.

There are two candidates for such enhanced transport, whose effects are being actively investigated. One is a deeply rooted fossil magnetic field, as advocated already by Mestel (1953), and more recently by Gough and McIntyre (1998). The problem with such a field is that it would inevitably connect with the convective envelope, and thus imprint its differential rotation on the radiative interior; this behavior was hinted in the stationary solutions obtained by Garaud (2002) and it has been confirmed more recently by Brun and Zahn (2005) through 3-dimensional time-dependent simulations. We are thus inclined to conclude that, in the Sun at least, it is not a magnetic field which is responsible for the uniform rotation of the radiative interior.

The alternative is presented by internal gravity waves (IGW). These are waves whose restoring force is the buoyancy force: they travel only in stably stratified regions, i.e. in radiation zones. Presumably, a whole spectrum of such waves is emitted at the base of the convection zone of late-type stars; they travel in the radiation zone, and they transport angular momentum which they deposit wherever they are dissipated through radiative damping. It is by shaping the rotation profile that they participate in the mixing of chemicals.

Let us first examine the behavior of those waves, of short wavelength, that are dissipated close to the convection zone. Prograde waves carry positive angular momentum, retrograde waves negative angular momentum. When they travel in a medium which is rotating faster then the region where they have been emitted, their frequency is Dopplershifted, leading to higher dissipation for the prograde waves than for the retrograde waves. For this reason the angular velocity tends to increase where it was already high, and its slope steepens until the shear becomes unstable. That turbulent layer then merges with the convection zone. But in the meanwhile the retrograde waves have deposited negative angular momentum somewhat further down, thus building there another shear layer of opposite direction, which now takes the place of the former one. And the cycle repeats. A similar phenomenon is observed in the Earth atmosphere, where it is called quasi-biennial oscillation (cf. McIntyre 2003). 


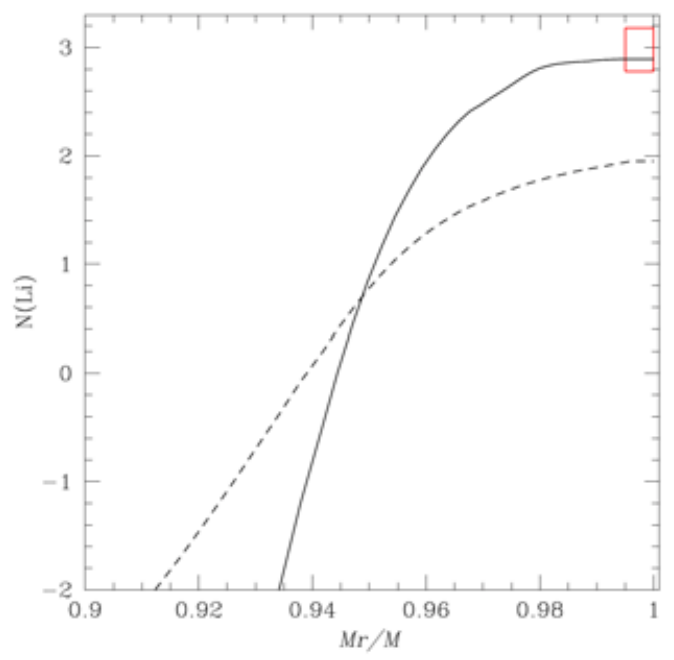

Figure 2. Profile of ${ }^{7} \mathrm{Li}$ in a $1.2 \mathrm{M} \odot$ star at the age of the Hyades (at the ZAMS it had an equatorial velocity of $50 \mathrm{~km} / \mathrm{s}$ ). The full line corresponds to the complete model, and the dashed line to the same model without IGW. The box indicates the observed Li abundance (Boesgaard \& King 2002). Taken from Talon \& Charbonnel (2005, courtesy A\&A).

The question then arises how this very thin shear layer, located just below the convection zone, affects the waves of longer wavelength, which are less dissipated. If the oscillation were perfectly symmetrical in time, its effect would be the same on the prograde and on the retrograde waves which traverse it. But if there is even a slight increase of $\Omega$ with depth in that layer, the prograde waves will be more dissipated, which allows the retrograde waves to extract angular momentum from the deep interior. This scenario has been established first through numerical simulations performed by Talon et al. (2002), using a rather crude approach where the Coriolis force was neglected and where an arbitrary turbulent viscosity was imposed. Recently it has been confirmed by Talon and Charbonnel (2004) through more detailed and more realistic calculations, applied specifically to Population II stars. Focusing on the differential properties of IGW with respect to stellar mass, they show that the wave flux varies little in the range of masses corresponding to the Spite plateau, and that it would probably lead to a quasi uniform rotation state, similar to that of the Sun. Work is under way to implement the full rotational mixing (circulation plus shear-induced turbulence) together with the gravity waves; it yields already excellent results for the Population I stars, such as reproducing the Li abundance of the Hyades, which is illustrated in fig. 2 (Talon \& Charbonnel 2005). And it is now being extended to metal-poor stars.

\section{Conclusion}

Let me conclude this brief presentation by insisting on the salient points.

- Due to gravitational settling, turbulent mixing and nuclear burning, the lithium abundance observed at the surface of any star decreases with age on the main-sequence; hence the value which is read in the Spite plateau is not the primeval one.

- In order to use this value to constrain the Big Bang Theory, one has to evaluate the amount of depletion, and for that one has to model not only the gravitational settling, but all mixing processes which operate in the star during its evolution. 
This is not an easy task, but considerable progress has been accomplished in recent years, particularly in calculating with higher precision the microscopic processes involved, and in recognizing and assessing the role of the internal gravity waves. The main weakness remains the description of the turbulent transport, as elsewhere in astrophysics. The approach of the physicist is to improve this modeling on sound physical grounds, rather than to lean on some parametric description adjusted to fit the WMAP result.

The famous plateau we owe to Monique and François is much less a puzzle nowadays, but it still represents a challenge!

\section{Acknowledgements}

I wish to thank Suzanne Talon for helping me to prepare this presentation, and for critically reading the manuscript.

\section{References}

Balachandran, S.C. 2002, Highlights of Astronomy (ASP), vol. 12, 276

Balachandran, S.C. \& Bell, R.A. 1997, Bull. American Astron. Soc., vol. 29, 1325

Bennett, C.L., Bay, M., Halpern, M. et al. 2003, ApJ 583, 1

Boesgaard, A.M. \& King, J.R. 2002, ApJ 565, 587

Boesgaard, A.M. \& Tripicco, M.J. 1986, ApJ 303, 724

Brun, A.S., Turk-Chièze, S. \& Zahn, J.-P. 1999, ApJ 525, 1032

Brun, A.S. \& Zahn, J.-P. 2005, A\&A (submitted)

Busse, F. 1982, ApJ 259, 759

Chaboyer, B. \& Zahn, J.-P. 1992, A\&A 253, 173

Deliyannis, C.P., Demarque, P., Kawaler, S.D. 1990, ApJS 73, 21

Eddington, A.S. 1925, Observatory 48, 78

Garaud, P. 2002, textitMNRAS 329, 1

Gough, D.O. \& McIntyre, M.E. 1998, Nature 394, 755

Maeder, A. \& Meynet, G. 2000, ARA\&A 38, 143

Maeder, A \& Zahn, J.-P. 1992, A\&A 334, 1000

McIntyre, M.E. 2003, in Stellar Astrophysical Fluid Dynamics, eds. M.J. Thompson \& J. Christensen-Dalsgaard (CUP), p. 111

Noerdlinger, P.D. \& Arigo, R.J. 1980, ApJ 237, L15

Mestel, L. 1953, MNRAS 113, 716

Michaud, G., Tarasik, D., Charland, Y., Pelletier, C. 1983, ApJ 269, 239

Pinsonneault, M.H., Kawaler, S.D., Sofia, S. \& Demarque, P. 1989, ApJ 338, 424

Proffitt, C.R. \& Michaud, G. 2001, ApJ 371, 584

Richard, O., Michaud, G., Richer, J. Turcotte, S., Turck-Chièze, S., VandenBerg, D.A. 2002, ApJ 568, 979

Ryan, S.G., Beers, T.C., Kajino, T., Rosolankova, K. 2001, ApJ 547, 231

Spergel, D.N., Verde, L., Peiris, H.V., et al. 2003, ApJS 148, 175

Spiegel, E.A. \& Zahn, J.-P. 1992, A\&\&A 265, 106

Stringfellow, G.S., Bodenheimer, P., Noerdlinger, P.D., Arigo, R.J. 1983, ApJ 264, 228

Sweet, P.A. 1950, MNRAS 110, 548

Talon, S. \& Charbonnel, C. 2004, A\&SA 418, 1051

Talon, S. \& Charbonnel, C. 2005, A\&A (in press) astro-ph/0505229

Talon, S., Kumar, P. \& Zahn, J.-P. 2002, ApJ 574, L175

Théado, S. \& Vauclair, S. 2001, A\&A 375, 70

Vauclair, S. 1999, A\&A 351, 973

Vauclair, S. \& Charbonnel, C. 1995, A\& A 295, 715

Vogt, H. 1925, Astron. Nachr. 223, 229

Zahn, J.-P. 1992, A\&SA 265, 115

Zahn, J.-P. 1994, A\&A 228, 829 